\title{
Optimal Sensor Placement for the Tower Structure Based on Key Compo- nents
}

\author{
Yang Chao-Shan, Cheng Hua* and Wang Zhong-Gang \\ Logistical Engineering University, ChongQing 401311, P.R. China
}

\begin{abstract}
In order to improve the large-scale complex spatial structure sensor placement, this paper puts forward an optimal sensor placement method for the tower structure based on key components. According to this method, different probabilities of the structure system's reliability degree caused by the same damage degree of components are firstly calculated; then component weight coefficients are introduced to measure the relative importance of various components in the whole structure system; the key components of the structure system are defined according to the value of weight coefficients; then modal parameters are adopted to analyze the damage sensitivity of the key components and to find out the vibration modes and measuring points sensitive to the damage of key components, thus to finish the sensor placement. Fully considering the structure characteristics, this method can solve the optimization issues, including the quantity of measurement points and the position, and avoid the complex iterative algorithm and modal expansion error, so it contributes to better controlling the structure state. Finally, through the calculation example of a communication tower, the feasibility and the validity of the method are proved.
\end{abstract}

Keywords: Key components, Sensitivity analysis, Tower structure, Sensor optimization.

\section{INTRODUCTION}

In order to effectively manage the large-scale complex structure and reduce the losses brought by disasters, structure health monitoring and damage testing have gradually become a research focus. Of them, sensor placement in the monitoring system can not only decide the effectiveness of the monitoring system, but can also directly influence the make or mar of the damage test. It is impractical to comprehensively gain the structure's response data through the sensor placement in large-scale complex spatial structure. Firstly, the quantity of sensors is limited, because too many sensors will not only lead to the increase of cost, but also can dramatically add to the complexity of the sensor system, and reduce the reliability of the system as well. Secondly, improper sensor placement cannot meet the demand of ideal parameter identification precision, and cause great burdens of data transmission, storage and treatment. Therefore, it is an important research topic for the optimal sensor placement through gaining adequate data and parameter identification precision through limited sensor quantity [1].

In recent years, many scholars have conducted a wide range of researches about the optimal placement of sensors, and have put forward lots of optimization methods [2-7], such as effective independence (EI) method, modal kinetic energy (MKE) method, QR decomposition method, mode reduction method, and genetic algorithm. Besides, modal

*Address correspondence to this author at the Logistical Engineering University, ChongQing 401311, P.R. China; E-mail: 402265413@qq.com assurance criteria (MAC), modified MAC (ModMac) and Fisher information matrix (FIM) are also put forward as the criteria to evaluate advantages and disadvantages of sensor placement. All these methods have their own limits, and their searching ability and reliability are not high. They are seldom applied to the practical large-scale complex spatial structure system [8].

As to the tower structure, it is characterized by complex structural forms, lots of bars, and great redundancy. Moreover, there are many secondary components, such as secondary web bar and partial tabula components, and the damage of secondary components have a slight influence on the structure's response parameters. To achieve the monitoring and data acquisition of the secondary components means more intensive placement and a greater number of sensors. The cost is undoubtedly huge. The damage of the structure's secondary components has a little influence on the structure's safety, so it is obvious that the monitoring and sensor placement of the secondary components means more losses than gains. Therefore, if placement methods effective to all structural damage are to be found through the modal parameters' quantity of structural damage without considering the possible damage of practical structures, and the importance of components, it will inevitably led to the increase of placement quantity and complexity.

Therefore, it is important to put forward an optimal sensor placement method for the tower structure based on the analysis of key components. Through calculating different degrees of decrease of structure failure probability caused by the same decrease degree of the resistance of various components, weight coefficients of components are introduced to 
measure the relative importance of various components in the structural system; the relative value of weight coefficients are adopted to select the key components; based on the damage information of the selected key components and the sensitivity analysis of modal vibration modes, the sensitivity value of different orders of vibration mode changes to the damage of key components is calculated, and the vibration modes and measuring points insensitive to the damage of key components are filtered to achieve an optimal sensor placement method of the structure. The method can avoid the errors brought by the modal expansion of the structure, and at the same time consider the influence of the damage of various components on the failure probability of the structures, achieve a differential treatment of the structural damage, improve the pertinence of sensor placement and enable the placement of limited sensors to gain a better control of the structure state. Finally, the feasibility and application prospects of the method are proved through the value calculation of a communication tower.

\section{KEY COMPONENTS}

After a period of use or suffering certain disasters, the structure may suffer different degrees of damage. The damage will definitely cause the changes of the components' resistance, which will further influence the safety and reliability of the structure. However, the influence of different components on the safety and reliability of the structure is different. Some are relatively important for having a greater influence on the reliability of the structure; and some are secondary for having a slight influence. In order to reflect the importance, the concept of the components' coefficients $[9,10]$ is introduced, and the weight coefficients are represented through the influence degree of certain damage on the structure system reliability. Obviously, the larger the components' weight coefficients, the larger the influence of the components on the structure system reliability and the more important the components are. Through proportionately reducing the resistance average value of certain component without changing the resistance of the remaining components, the increase of failure probability of the structure system is calculated. Based on that, the weight coefficients of various components are worked out. The key components which have a greater influence on the structure's reliability were found out through calculating the weight coefficients of various components.

Calculation of the importance of the $j$ component:

$$
f(j)=\lim _{\Delta \mu_{R 0}(j) \rightarrow 0} \frac{\Delta p_{f 0}(j)}{\Delta \mu_{R 0}(j)}, j=1,2, \cdots, n_{s}
$$

Where, $n_{s}$ stands for the total quantity of components in the structure system, $\Delta \mu_{R 0}(j)$ for the reduction value of the $j$ component compared with its initial resistance average value, $\Delta p_{f 0}(j)$ for the increase value of the structure system failure probability caused by the change of $j$ component's average value by $\Delta \mu_{R 0}(j)$, and $f(j)$ for the influence degree of the reduced component's resistance average value on the structure system failure probability. Thus, the weight coefficient of the component, $A(j)$, can be gained through the following formula:

$$
A(j)=\frac{f(j)}{\sum_{k=1}^{n_{s}} f(k)}, j=1,2, \cdots, n_{s}
$$

From formula (1) and (2), it can be known that the failure probability of the structure system before and after the reduction of the component's resistance must be worked out so as to gain the component's weight coefficients. With the rapid development of computers and large-scale software of finite element analysis, the calculation of the structure system failure probability through Monte Carlo algorithm has been widely applied. According to the method, the nonlinearity of performance function and the curved surface complexity of the ultimate state needn't have to be considered, and the analysis is only confined to the precision of mechanical analysis and the sample size. Moreover, the method boasts simple and clear thinking and accurate analysis result, thus is often employed to verify the other methods. Through the sampling of random variable, the Monte Carlo method generates lots of sample values of random variables. By making use of these sample values, and placing them in the performance function, it can judge the structure state. After a statistical analysis of lots of calculation results, it uses occurrence frequency to estimate the failure probability or reliability of the structure. The expression is shown as below:

$$
\hat{P}_{f}=\frac{1}{N} \sum_{i=1}^{N} I\left[g_{X}\left(X_{1}^{i}, X_{2}^{i}, \cdots X_{n}^{i}\right)\right]
$$

Where, $X_{k}(k=1,2, \cdots, n)$ stands for $n$ independent random variables of the performance function, the superscript $i$ for the $i$ sampling, $N$ for the total number of samplings, " $\wedge$ " for the sampling value adopted, and $I[]$ for the indicative function:

$$
\begin{aligned}
& I\left[g_{X}\left(X_{1}^{i}, X_{2}^{i}, \cdots X_{n}^{i}\right)\right]=0, g_{X}\left(X_{1}^{i}, X_{2}^{i}, \cdots X_{n}^{i}\right) \geq 0 \\
& I\left[g_{X}\left(X_{1}^{i}, X_{2}^{i}, \cdots X_{n}^{i}\right)\right]=1, g_{X}\left(X_{1}^{i}, X_{2}^{i}, \cdots X_{n}^{i}\right)<0
\end{aligned}
$$

In the large-scale finite element software, ANSYS, Prob Design with reliable structure provides three Monte Carlo Sims, namely direct sampling, Latin sampling and user defined sampling. ANSYS Parametric Design Language (APDL) is adopted to compile the structure model, subnetting, loading and other command streams for the establishment of structure analysis model. By employing the Monte Carlo analysis method of ANSYS and probability design system module's stochastic simulation and statistical analysis functions, the calculation of structure failure probability or reliability can be realized. 
Thus, the key components can be achieved through the following steps:

(1) Set the initialized data, including the average value and variable coefficients of components' scale, resistance, load and other random variables;

(2) Establish the model and calculate the failure probability of the structure system $P_{f}$;

(3) Proportionately reduce the resistance of $j$ unit component, and maintain the other components unchanged; repeat Step (2) and calculate the failure probability, $P_{f 0}(j)$, corresponding to the structure system; and work out the increase value of the structure system failure probability caused by the decrease of the resistance of the $j$ unit component $\Delta p_{f 0}(j)=P_{f 0}(j)-P_{f}$;

(4) Put the formula above into the formula to calculate the importance of components and the weight coefficients of various components;

(5) Arrange the structure components according to their priority and the components' weight coefficients, and select the key components that suffer a greater influence from the structural damage through setting the proper penalty value.

\section{OPTIMAL SENSOR}

Structural damage can cause changes of physical properties, including mass, stiffness and damping, thus causing corresponding changes of modal parameters (frequency and vibration mode). The health monitoring and damage detecting of the structure achieve the collection of structural modal parameters through the sensor placement, and achieve the control of structure's actual state through monitoring the corresponding relationship between structure's modal parameters and physical parameters.

The key components have a greater influence on the structure system reliability, so these components are comparatively important. It is necessary to place the sensors in a targeted way so as to prevent the occurrence of possible damage of key components. Based on that, this paper conducts sensitivity analysis of the modal parameter changes caused by key components [11] to find out the vibration modes and measuring points sensitive to the damage of the key components, and monitor the key components through sensor placement in the sensitive vibration modes and measuring points.

To a structure system with $n$ degree of freedom, the structure's vibration equation can be expressed as below:

$$
(K-\lambda M) \Phi=0
$$

Where, $\boldsymbol{K}$ and $\boldsymbol{M}$ stands for stiffness matrix and mass matrix of the structure respectively, and $\lambda$ and $\Phi$ for characteristic matrix and modal vibration mode matrix of the structure respectively.
It is assumed that structure damage cannot cause the mass changes, and when the structure suffers little damage, the structure vibration control equation is shown as below:

$[(K-\Delta K)-(\lambda-\Delta \lambda) M](\Phi-\Delta \Phi)=0$

Where, $\Delta \boldsymbol{K}, \Delta \boldsymbol{\lambda}$ and $\Delta \boldsymbol{\Phi}$ stand for the slight changes of stiffness, characteristic value and modal vibration mode matrix caused by the structural damage. Ignoring the higher order indefinite small, the above equation can be summarized as:

$(K-\lambda M) \Delta \Phi=\Delta \lambda M \Phi-\Delta K \Phi$

Where, the vibration mode vector and characteristic value meets the following equation:

$\left(K-\lambda_{i} M\right) \Delta \Phi_{i}=\Delta \lambda_{i} M \Phi_{i}-\Delta K \Phi_{i}$

Where, the change of the vibration modes can be expressed as below through the linear combination of the initialized system modals:

$$
\Delta \Phi_{i}=\sum_{l=1}^{n} \delta_{i l} \Phi_{l}
$$

Where, $\Delta \Phi_{i}$ stands for the variation of $i$ order vibration mode, $\Phi_{l}$ for the $l$ order vibration mode and $\delta_{i l}$ for the vibration change parameters and coefficients of $l$ order vibration mode.

Put (10) into (9) and use $\Phi_{l}^{\mathrm{T}}$ for the pre-multiplication of the two sides of the equation:

$$
\sum_{l=1}^{n} \delta_{i l} \Phi_{l}^{\mathrm{T}}\left(\boldsymbol{K}-\lambda_{i} \boldsymbol{M}\right) \Phi_{l}=\Delta \lambda_{i} \Phi_{l}^{\mathrm{T}} \boldsymbol{M} \Phi_{i}-\Phi_{l}^{\mathrm{T}} \Delta \boldsymbol{K} \Phi_{i}
$$

According to the orthogonality principle of the vibration mode to the stiffness matrix and the quality matrix, the following result is gained:

$$
\begin{cases}\Phi_{l}^{\mathrm{T}} \boldsymbol{K} \Phi_{l}=0 & \Phi_{l}^{\mathrm{T}} \boldsymbol{K} \Phi_{l}=\lambda_{l} \\ \boldsymbol{\Phi}_{l}^{\mathrm{T}} \boldsymbol{M} \Phi_{l}=0 & \boldsymbol{\Phi}_{l}^{\mathrm{T}} \boldsymbol{M} \Phi_{l}=\boldsymbol{I}\end{cases}
$$
(11):

When $l \neq i$, the following result can be gained based on

$$
\delta_{i l}=\frac{-\Phi_{l}^{\mathrm{T}} \Delta K \Phi_{i}}{\lambda_{l}-\lambda_{i}}
$$

When $l=i$, and $\delta_{i l}=0$, the following equation can be gained:

$$
\Delta \Phi_{i}=\sum_{\substack{l=1 \\ l \neq i}}^{n} \frac{-\Phi_{l}^{\mathrm{T}} \Delta K \Phi_{i}}{\lambda_{l}-\lambda_{i}} \Phi_{l}
$$




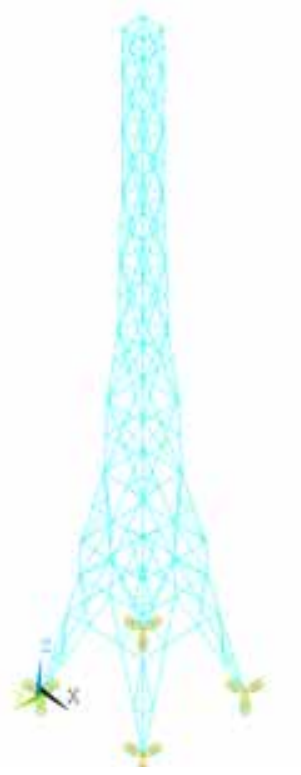

Fig. (1). Model of tower structure.

The changes of the structure's stiffness matrix can be expressed as below through the changes of the unit stiffness:

$\Delta \boldsymbol{K}=\sum_{k=1}^{n} \Delta \alpha_{k} \boldsymbol{K}_{k}$

Where, $\Delta \alpha_{k}$ stands for the damage coefficient of the $k$ unit, and $\boldsymbol{K}_{k}$ for the unit stiffness matrix of the whole coordinate system.

Put (15) into (14), and the following result can be gained:

$\Delta \Phi_{i}=\sum_{k=1}^{n}\left\{\Delta \alpha_{k} \sum_{\substack{l=1 \\ l \neq i}}^{n} \frac{-\Phi_{l}^{\mathrm{T}} \boldsymbol{K}_{k} \Phi_{i}}{\lambda_{l}-\lambda_{i}} \Phi_{l}\right\}=\sum_{k=1}^{n} \Delta \alpha_{k} \boldsymbol{S}_{i k}$

$\boldsymbol{S}_{i k}=\left\{\begin{array}{rr}\sum_{\substack{l=1 \\ l \neq i}}^{n} \frac{-\Phi_{l}^{\mathrm{T}} K_{1} \Phi_{i}}{\lambda_{l}-\lambda_{i}} \Phi_{l}, \sum_{\substack{l=1 \\ l \neq i}}^{n} \frac{-\Phi_{l}^{\mathrm{T}} \boldsymbol{K}_{2} \Phi_{i}}{\lambda_{l}-\lambda_{i}} \Phi_{l}, \\ \cdots, \sum_{\substack{l=1 \\ l \neq i}}^{n} \frac{-\Phi_{l}^{\mathrm{T}} K_{n} \Phi_{i}}{\lambda_{l}-\lambda_{i}} \Phi_{l}\end{array}\right\}$

Assuming in turn that the $k$ unit among the key components suffers damage, and the damage coefficient is $\Delta \alpha_{k}=0.1$, and maintain the other units suffering no damage, $\Delta \Phi_{i}$ can be gained through ANSYS, and the following formula is gained through (16):

$S_{i k}=\frac{\Delta \Phi_{i}}{\Delta \alpha_{k}}$

Where, $S_{i k}$ stands for the sensitivity of $i$ order vibration mode to the damage of $k$ unit. Then $m$ order modal vibration mode which is sensitive to the damage of $k$ unit is cho-

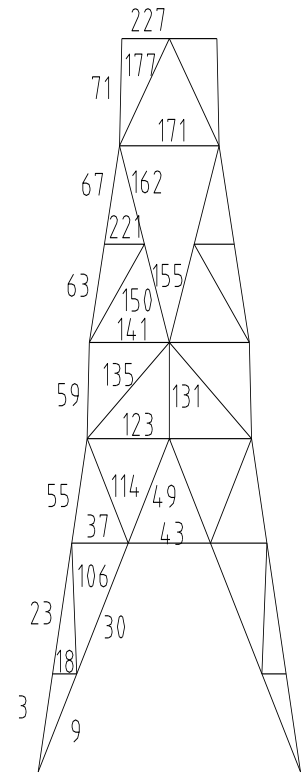

Fig. (2). Rod number the bottom section.

sen, and the damage sensitivity factor matrix of the $k$ unit can be gained:

$$
S_{k}=\sqrt{\sum_{m} S_{m k}}
$$

Use a proportion coefficient reflecting the contribution percentage of high order mode to modify the sensitivity factor matrix, and the proportion coefficient can be worked out through the following formula:

$$
\xi_{k}=\omega_{k j} / \sum_{j=1}^{20} \omega_{k j}
$$

Where, $\omega_{k j}$ stands for the $j$ order frequency of $k$ unit's damage and $\xi_{k}$ for the proportion coefficient matrix of the $k$ unit's damage.

\section{NUMERICAL VERIFICATION}

This paper takes a communication tower as an example. The tower is $30 \mathrm{~m}$ high, made up of five kinds of sectional angle iron. The total quantity of bars is 316 . The simulation is finished through the adoption of spatial bars and ANSYS. The finished model is shown in Figs. (1-4). The field type is $B$, and the fundamental wind pressure and bar yield strength are both in line with the normal distribution. Their average value is $\mu_{w 0}=0.45 \mathrm{KN} / \mathrm{m}^{2}$ and $\mu_{\sigma}=235 \mathrm{~N} / \mathrm{mm}^{2}$ respectively. The variable coefficients are set as 0.3 and 0.1 respectively, and parametric modeling and Monte Carlo method are adopted to calculate the structure failure probability under the largest designed wind load at $45^{\circ}$. The analysis of the structure's whole reliability adopts three indexes, namely largest structural displacement, component's maximum stress and component's buckling failure. 


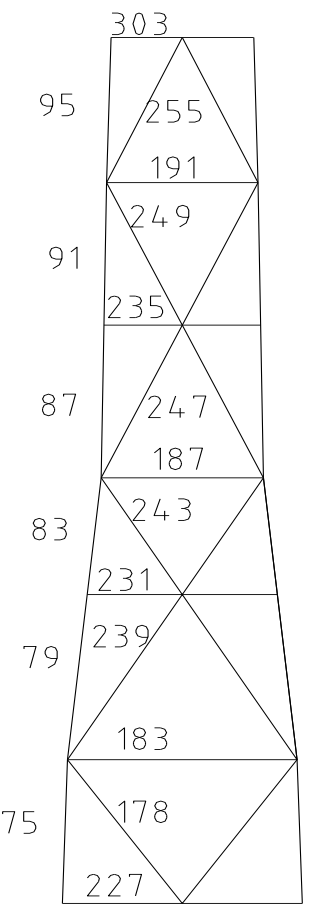

Fig. (3). Rod number of the central section.

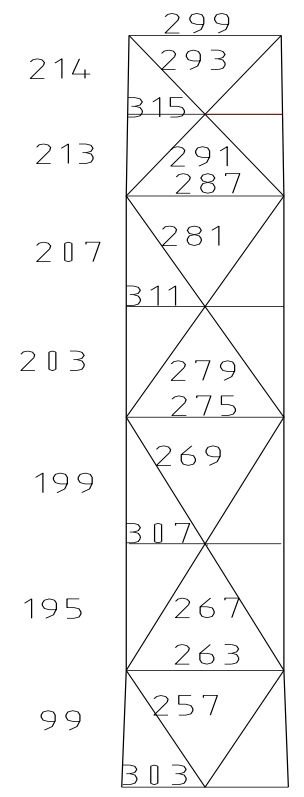

Fig. (4). Rod number of the top section.

The calculation finds out that the occurrence probability of component's buckling failure is far larger than that of the other two indexes, which suggests that the damage of tower structure is mainly controlled by the component's buckling failure, which coincides with the project in which buckling failure is common to see in tower structure. By making use of the symmetry of the tower structure, regarding the component's buckling failure as the index and selecting one eighth of the quantity of tower bars to correspond to the angle bars under the wind load function at $45^{\circ}$, the weight coefficient of the component's importance is calculated (See Table 1).

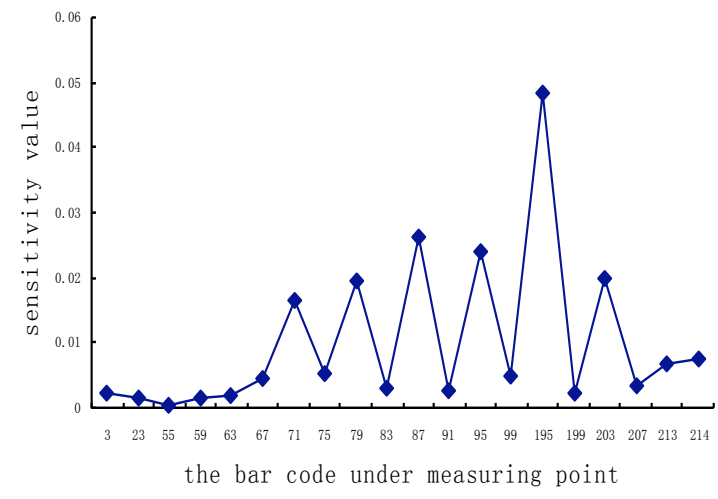

Fig. (5). Sensitive curve of $X$ direction.

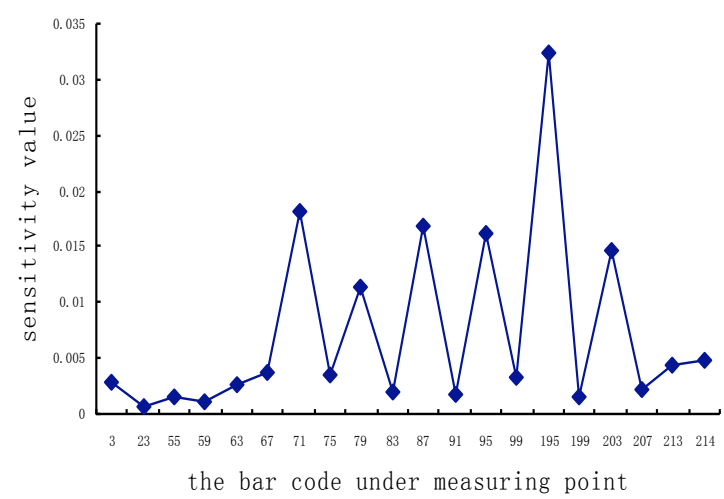

Fig. (6). Sensitive curve of Y direction.

It can be seen from Table 1 that: 1) the coefficients of chord bars are twice higher than those of diagonal web bars, which suggests that chord bars have the greatest influence on the structure's failure probability, following by diagonal web bars, and the horizontal bars the smallest. This is in line with the concept in project design that chord bars are the most important and the horizontal bars the weakest; 2) the weight coefficients of the chord bars in the deformation area of the structure's cross-section is the largest, which suggests that chord bars in the variable cross-section has the largest influence on the reliability of the structure. This is in line with the practical project situations that deformations are frequent to happen in the cross-section in the tower structure. Obviously, to enhance the health monitoring and damage testing of the key components is of vital importance to ensure the reliability of the results. To the end, this paper mainly selects eight chord bars near the cross-section as the key components to conduct the calculation of optimal sensor placement.

Due to the influence of the environment and the measurement noise in the practical project testing, it is comparatively difficult to test the high order vibration. Therefore, modes of the previous 7 orders are mainly adopted for the numerical modeling. Then the elasticity modulus of the key components is reduced by $20 \%$ so as to monitor simulate the components' damage. The $m$ order modal vibration mode 
Table 1. Weight coefficient of element.

\begin{tabular}{|c|c|c|c|c|c|}
\hline \multicolumn{2}{|c|}{ Chord Bars } & \multicolumn{2}{c|}{ Diagonal Web Bars } & \multicolumn{2}{c|}{ Horizontal Bars } \\
\hline Number & Weight Coefficient & Number & Weight Coefficient & 43 & 183 \\
\hline 59 & $7.930 \mathrm{E}-2$ & 114 & $3.324 \mathrm{E}-2$ & 141 & $4.038 \mathrm{E}-4$ \\
\hline 67 & $7.928 \mathrm{E}-2$ & 30 & $3.215 \mathrm{E}-2$ & 123 & $5.782 \mathrm{E}-5$ \\
\hline 63 & $7.914 \mathrm{E}-2$ & 9 & $2.287 \mathrm{E}-2$ & 221 & $2.783 \mathrm{E}-5$ \\
\hline 75 & $7.327 \mathrm{E}-2$ & 243 & $1.046 \mathrm{E}-2$ & $1.045 \mathrm{E}-2$ & 171 \\
\hline 71 & $7.312 \mathrm{E}-2$ & 239 & $8.921 \mathrm{E}-3$ & $3.930 \mathrm{E}-5$ \\
\hline 83 & $7.252 \mathrm{E}-2$ & 177 & 178 & $8.903 \mathrm{E}-3$ & $3.816 \mathrm{E}-6$ \\
\hline 79 & $7.241 \mathrm{E}-2$ & 135 & $\ldots .034 \mathrm{E}-3$ & & 18 \\
\hline
\end{tabular}

sensitive to the damage of various components is selected to calculate the sensitivity value of various components.

The low-order modalities usually can reflect the general information of the response structure. High-order modality can gain structure's partial response and is sensitive to the partial damage. According to the modal sensitivity value, it can be found that the top three order vibration modes most sensitive to the damage of the key components are the fifth order, the sixth order and the seventh order vibration order in turn. This suggests that it is feasible to filter the low-order modalities through the largest sensitivity vale and maintain the high-order modality which can reflect the information of partial damage. Fig. (5) and Fig. (6) provide the sensitivity value curve of some key components through the calculation of sensitive modalities. The wave crest of the curve is the quantity of the sensors required, and the position of the wave crest is the placement point of the sensors.

The tower structure boasts strong spatial symmetry, and the modal changes on the $\mathrm{X}$ direction and $\mathrm{Y}$ direction caused by the damage of chord bars are the same. Therefore, the calculation result of the sensitivity values and the placement of the measuring points of the sensors should be symmetric. The wave crest quantity and the wave crest position in Fig. (5) and Fig. (6) are in line with each other, which better meets the requirement of the characteristic of the tower structure.

\section{CONCLUSION}

Through calculating the weight coefficients of the components, and analyzing the parametric sensitivity, this paper put forward an optimal sensor placement method for the tower structure based on the key components. Its main advantages include:

(1) This paper considers the influence degree of the damage of various components on the structure's failure probability, achieves the differentiated treatment of the structure components' damage, and makes the sensor placement more targeted;

(2) The employment of sensitivity analysis can not only achieve the filtration of the modal information which is insensitive to damage, but can also achieve the optimal placement of the sensors' quantity and position;

(3) The analysis of the key components and the sensor placement takes a full consideration of the primitive structural characteristics, and the result of the key components' analysis and the sensor placement is in line with the practical projects. Moreover, the method is based on simple principles, but boasts strong operability and great application prospects.

\section{CONFLICT OF INTEREST}

The authors confirm that this article content has no conflict of interest.

\section{ACKNOWLEDGMENTS}

This work is supported by the Key Project of Guangxi Social Sciences, China (No.gxsk201424), the Education Science fund of the Education Department of Guangxi, China (No.2014JGA268).

\section{REFERENCES}

[1] T. Yi, H. Li, and M. Gu, "Research on optimal sensor placement of GuangZhou new TV tower based on model reduction", Engineering Mechanics, vol. 29, no. 3, pp. 55-61, 2012

[2] W. Liu, W. Gao, and H. Li, "Improved optimal sensor placement methods based on effective independence", Journal of Vibration and Shock, vol. 32, no. 6, pp. 54-61, 2013

[3] T. Yi, X. Zhang, and H. Li, "Research on optimal sensor placement based on improved monkey algorithm", Chinese Journal of Computational Mechanics, vol. 30, no. 2, pp. 218-223, 2013 
[4] C. Zhou, D. Li, and H. Li, "Optimal sensor placement for structural modal test", Journal of Vibration Engineering, vol. 27, no. 1, pp. 84-90, 2014

[5] X. Sun, X. Feng, and J. Zhou, "Optimal sensor placement for health monitoring of latticed shell structure", Chinese Journal of Computational Mechanics, vol. 27, no. 3, pp. 482-488, 2010.

[6] J. Cheng, W. Yan, and Y. Chen, "Optimal sensor placement for bridge structure based on improved effective independence", Journal of Vibration, Measurement \& Diagnosis, vol. 32, no. 5, pp. 812-816, 2012.

[7] D. Xu, "Studies on structural damage detection and optimal sensor placement", Chongqing: Chongqing University, 2011.
[8] S. Jiang, B. Yang, and Z. Wu, "Sensor arrangement method based on vulnerability analysis for a truss bridge", Engineering Mechanics, vol. 27, pp. 263-265, 2010.

[9] H. Rong, and J. Ma, "Study on component weighting factors in structural reliability evaluation", Journal of Xi'An Jiao Tong University, vol. 35, no. 12, pp. 1300-1304, 2001.

[10] Z. Luo. Reliability analysis of truss bridge structural system and recognition of component importance, Guangzhou: South China University of Technology, 2010.

[11] Z. Wu, Y. He, and X. Jian, "Optimal sensor placement based on sensitivity anslysis of structural damage", Engineering Mechanics. vol. 26, no. 5, pp. 239-244, 2009.

Received: September 22, 2014

Revised: November 03, 2014

Accepted: November 06, 2014

(C) Chao-Shan et al.; Licensee Bentham Open.

This is an open access article licensed under the terms of the Creative Commons Attribution Non-Commercial License (http://creativecommons.org/licenses/by-nc/3.0/) which permits unrestricted, non-commercial use, distribution and reproduction in any medium, provided the work is properly cited. 